\title{
Restricted diets - are they nutritionally adequate?
}

\author{
L.A. Bush and K.E. Lane \\ ${ }^{1}$ School of Sport Studies, Leisure and Nutrition, Liverpool John Moores University, Liverpool, United Kingdom, \\ $L 176 B D$
}

Diets labelled as fad or restrictive are becoming increasingly fashionable with adult women in the 20-60 age bracket being more likely to engage in a restricted $\operatorname{diet}^{(1)}$. A diet is classed as 'restrictive' if it promises dramatic, short-term weight loss and severely restricts certain food groups ${ }^{(2)}$. There is increasing concern that restricted diets may not be providing adequate levels of micronutrients $^{(3,4)}$. Three restricted diets that have grown in popularity in recent years are the Palaeolithic (Paleo), Alkaline and Montignac diets $^{(5)}$. Despite the weight loss and some beneficial health effects associated with these diets, little is known about their micronutrient status in terms of whether they satisfy official dietary recommendations. The aim of this study was establish whether these three restricted diets satisfy the current UK Reference Nutrient Intake (RNI) micronutrient recommendations for female adults.

Three daily menus from each of these diets were evaluated for micronutrient content using McCance and Widdowsons (MCW) food composition data from the Dietplan 7 nutritional software programme. An average micronutrient intake was calculated and compared to the official UK Reference Nutrient Intakes (RNI) guidelines for women. SPSS (V24) was used to perform data analysis with independent t-tests used to compare mean micronutrient intakes against the RNI and the ANOVA test used to determine comparisons between the diets.

Intakes of vitamin D were significantly below the RNI for all three diets $(p \leqslant 0.05)$ with calcium also being below the RNI for the Paleo and Alkaline diets and iron below the RNI for the Montignac and Alkaline diets although not significantly ( $p \geqslant 0.05)$. The Alkaline diet also had intakes significantly below the RNI for the minerals selenium and iodine (p $\leqslant 0.05)$ and for vitamins B2 and $\mathrm{B} 12$ but not significantly $(\mathrm{p} \geqslant 0.05)$. Adult females who adhere to a restricted diet for long periods may be putting themselves at risk of micronutrient deficiency. Health professionals need to ensure the information regarding restricted diets is communicated effectively by emphasising the potential nutritional pitfalls.

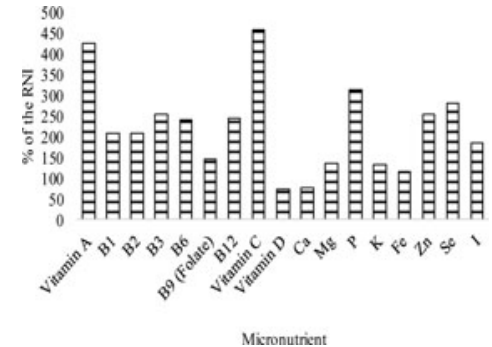

Fig. 1. Micronutrient intakes as a percentage of the RNI for the Paleo diet.

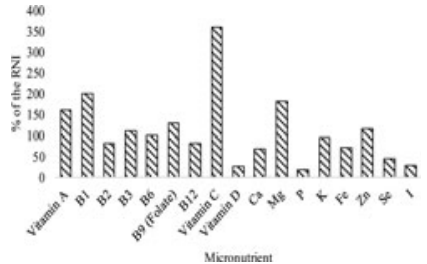

Fig. 2. Micronutrient intakes as a percentage of the RNI for the Alkaline diet.

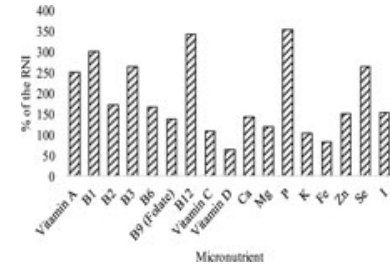

Fig. 3. Micronutrient intakes as a percentage of the RNI for the Montignac diet.

1. Clifford E (2012) Diet trends amongst women. www.mintel.com/diet-trends.

2. Crowe T (2008) Nutridate 19(2), pp. 1-4.

3. Cioffi I, Santarpia L, et al. (2014) Nutritional Therapy \& Metabolism 32(3), 112-120.

4. Richman A (2016) Fad Diet or Healthy Trend? www.progressivegrocer.com.

5. Hooper B (2014) Nutrition Bulletin 39, 284-278. 